\section{HEALTHY LIFESTYLE INTERVENTION FOR OBESE CHILDREN AND THEIR FAMILIES AS A PART OF THE PREVENTIVE HEALTH CARE PROGRAMME FOR CHILDREN AND ADOLESCENTS}

Matjaž Homšak*, P Truden-Dobrin, B Vogrin, P Kotnik, T Pibernik. Health Care Center Lenart

10.1136/archdischild-2021-europaediatrics.69

Goal Childhood obesity is one of the most serious public health challenges of the 21st century. An increase in childhood obesity has been observed in most countries. In Slovenia, childhood obesity and lower physical fitness is an important public health problem. Therefore, a healthy lifestyle intervention was developed and piloted at the primary health care level for obese children 8 and 11 years old and their families. The aim was also to strengthen the cooperation of the local health care services with schools and local community to support children and their families in lifestyle change.

Method A pilot intervention was performed in 2018 and 2019 in 25 Health Promotion Centres in Slovenia (27 Health care centres at the primary level).

The primary care paediatrician led and coordinated the team that carried out the intervention. The team included a psychologist, a dietitian, a kinesiologist and a nurse. A detailed information was collected and a plan was prepared for a child and the family and the goals of the intervention were set. Intervention consisted of individual sessions for a family with the team or a particular member of the team and group sessions that focused on physical activity, healthy diet, motivation, sleep, the use of electronic devices and social media. There were workshops and practical sessions for the families or in parallel for children and for parents separately.

The team coordinated with the school staff regarding school meals and additional physical activity and also with the local community to ensure there were facilities and activities for the family in the local community.

The role of local sport clubs and societies was particularly important during the summer holidays to ensure active and healthy lifestyle and motivation for change.

Focus groups method was used to evaluate the acceptability of the intervention and the extent to which the new intervention might meet the needs of the target population and organizational setting where it was carried out. Focus groups with parents were conducted. Focus groups lasted approximately 60 minutes and explored factors that made intervention acceptable to parents and their children. Focus groups with health professional were also conducted to explore their views together with discussions on preferred methods of work. Focus groups were audio-recorded, transcribed verbatim and then analysed.

Results The results from series of focus groups showed what intervention components were most acceptable to the parents and how they might best be implemented.

Conclusion The piloted intervention was acceptable to parents and health professionals. Information from focus groups should guide the design and development of lifestyle interventions.

\section{TRICHOBEZOAR: THE IMPORTANCE OF A MULTIDISCIPLINARY APPROACH}

Catarina Macedo Francisco*, Pedro Pereira, Íris Santos Silva, Pedro Guerra, Isabel Gonzalez, António P Pissarra. Sousa Martins Hospital

\subsection{6/archdischild-2021-europaediatrics.70}

Introduction Abdominal pain is one of the most frequent causes of pediatric Hospital admission, and therefore is frequently overlooked; its cause is usually attributed to cramps or constipation.

Nevertheless, a thorough investigation should always be performed.

Case Study 14 year-old girl, institutionalized, admitted to the Emergency Department due to abdominal pain, in the context of constipation, which was already known.

The patient has a history of Psychiatric disease, and is taking Risperidone $0.5 \mathrm{mg}$ once daily.

The patient did an abdominal radiography that showed abdominal distension and presence of a large amount of feces.

After bowel enema, the pain persisted, and due to the recurrent symptoms, she was hospitalized.

Her blood tests demonstrated only Anemia (Hb: 10g/dL; MCV: $74.7 \mathrm{fL} ; \mathrm{MCH}$ :

$20.9 \mathrm{pg}$; CHCM: $27.9 \mathrm{~g} / \mathrm{dL}$ ), low total proteins: $5.18 \mathrm{~g} /$ $\mathrm{dL}$; and low Albumin: $3.17 \mathrm{~g} / \mathrm{dL}$.

Her abdominal pain was progressively increasing, and she then performed an abdominal CT scan, which showed a large mass in the stomach extending up to the pylorus, with extraabdominal gas adjacent to the gastric antrum. The hypothesis of trichobezoar with gastric perforation was primarily considered. A small pleural effusion on the left side was also described.

Surgical approach was performed and a large trichobezoar, of about $20 \mathrm{~cm}$, was successfully removed. Anterior stomach perforation and chemical peritonitis was also found.

After surgery, the patient then admitted to suffer from Tricophagy, as a part of her Psychiatric condition.

During recovery, the pleural effusion increased in size, despite intravenous albumin therapy and drainage was performed. She was then discharged to her institution after a week.

Currently, her bowel movement is completely restored, and she is taking iron oral supplements.

She maintains follow-up at our outpatient clinic, maintaining slight chest pain of pleuritic character.

Conclusion Among adolescents, the frequency of psychosomatic symptoms is common. Nevertheless, and as we can see in this case, sometimes the etiology of abdominal pain can be quite serious, and even fatal if left untreated.

We should always value the overall clinical state of the patient in order to guide our medical approach. The collaboration between Pediatrics, Radiology and General Surgery was vital for a successful diagnosis and treatment. 